Mathematical Modelling and Analysis

Volume 17 Number 5, November 2012, 642-649

http://dx.doi.org/10.3846/13926292.2012.734865

(c) Vilnius Gediminas Technical University, 2012
Publisher: Taylor\&Francis and VGTU

http://www.tandfonline.com/TMMA

Print ISSN: 1392-6292

Online ISSN: 1648-3510

\title{
Estimation of Turning Point Location of Convex Solutions for Nonlinear Second-Order Differential Equations
}

\section{Robert Vrabel}

Slovak University of Technology in Bratislava, Faculty of Materials Science and Technology

Hajdoczyho 1, 91701 Trnava, Slovakia

E-mail: robert.vrabel@stuba.sk

Received December 16, 2011; revised August 24, 2012; published online November 1, 2012

\begin{abstract}
In this paper, we study the existence and location of turning points of the convex solutions for a certain class of the ordinary differential equations subject to the Dirichlet boundary conditions. We propose a practical and effective method for calculating the lower and upper bounds of turning point location.
\end{abstract}

Keywords: boundary value problem, second-order equation, nonlinear differential equation.

AMS Subject Classification: $34 \mathrm{~B} 15 ; 34 \mathrm{C} 11 ; 34 \mathrm{~A} 40$.

\section{Introduction}

We consider the following nonlinear second-order Dirichlet boundary value problem

$$
\begin{gathered}
y^{\prime \prime}+f\left(x, y, y^{\prime}\right)=0, \quad x \in[a, b] \\
y(a)=A, \quad y(b)=B .
\end{gathered}
$$

In this paper, $f \in C\left([a, b] \times \mathbb{R}^{2}\right)$ is a negative-valued function and $A, B$ are the real numbers. We note that from the assumption $f<0$ it follows that the solution of problem $(1.1),(1.2)$ is a convex function on $[a, b]$.

We focus our attention on the existence and an estimate of location of so -called turning points $x_{T P}$ of the solutions $y$ of the problem (1.1), (1.2). We define a turning point of a solution $y$ as the point $x_{T P} \in(a, b)$ such that $y^{\prime}\left(x_{T P}\right)=0$ and $y^{\prime \prime}\left(x_{T P}\right) \neq 0$. Obviously, under our assumption, there exists at most one turning point $x_{T P}$ of solution $y$ and $y^{\prime \prime}\left(x_{T P}\right)>0$, that is, $x_{T P}$ is a point of local minimum of the solution $y$. The problem of computing a value $x_{T P}$ for which a solution $y$ (or other function) has a turning point occurs frequently in scientific work. In many cases of practical interest, however, an analytic form for $y$ or $y^{\prime}$ is unavailable or difficult to obtain. 
The problem of existence and uniqueness of concave and convex solutions for third-order autonomous differential equation arising in the study of the mixed convection boundary layer flow along a semi-infinite vertical plate embedded in a saturated porous medium was examined in [1].

To our knowledge, no paper exists addressing the question of turning points location of the solutions for second-order equations directly from differential equations.

Boundary value problems (1.1), (1.2) can also arise in the study of the steady-states of a heated or cooled bar with a thermostat described by a scalar partial differential equation

$$
\frac{\partial y}{\partial t}=\frac{\partial^{2} y}{\partial x^{2}}+f\left(x, y, \frac{\partial y}{\partial x}\right)
$$

with stationary condition $\partial y / \partial t=0$, where the controller at $x=b$ maintains a temperature according to the temperature registered by a sensor at $x=$ $a$. In this case, we consider a uniform bar of length $b-a$ with non-uniform temperature lying on the $x$-axis from $x=a$ to $x=b$ and the temperature $B$ on the right end of bar is a function of the temperature $A$ on the left end of bar, $B=\phi(A)$; the point $x_{T P}$, if one exists, is the minimum temperature point on the considered bar.

\section{Necessary and Sufficient Condition for the Existence of Turning Point}

Now we derive a necessary and sufficient condition for the existence of turning point for solution of problem (1.1), (1.2).

Theorem 1. The point $x_{T P} \in(a, b)$ is a turning point of the solution $y$ of the problem (1.1), (1.2) if and only if the integral identity

$$
(b-a) \int_{a}^{x_{T P}} f\left(s, y(s), y^{\prime}(s)\right) \mathrm{d} s=(B-A)+\int_{a}^{b} f\left(s, y(s), y^{\prime}(s)\right)(b-s) \mathrm{d} s
$$

holds.

Proof. Let $y$ be the solution of the problem (1.1), (1.2). Integrating (1.1) twice with respect to the independent variable between $a$ and $x$ we obtain

$$
\begin{aligned}
y^{\prime}(x) & =y^{\prime}(a)-\int_{a}^{x} f\left(s, y(s), y^{\prime}(s)\right) \mathrm{d} s, \\
y(x) & =A+y^{\prime}(a)(x-a)-\int_{a}^{x}\left(\int_{a}^{z} f\left(s, y(s), y^{\prime}(s)\right) \mathrm{d} s\right) \mathrm{d} z .
\end{aligned}
$$

From (2.3) we have

$$
y^{\prime}(a)=\frac{1}{b-a}\left(B-A+\int_{a}^{b}\left(\int_{a}^{z} f\left(s, y(s), y^{\prime}(s)\right) \mathrm{d} s\right) \mathrm{d} z\right) .
$$


Putting (2.4) into (2.2) we get

$$
\begin{aligned}
y^{\prime}(x)= & \frac{1}{b-a}\left(B-A+\int_{a}^{b}\left(\int_{a}^{z} f\left(s, y(s), y^{\prime}(s)\right) \mathrm{d} s\right) \mathrm{d} z\right) \\
& -\int_{a}^{x} f\left(s, y(s), y^{\prime}(s)\right) \mathrm{d} s .
\end{aligned}
$$

Equating (2.5) with zero, which is a necessary and sufficient condition for the existence of a turning point $x_{T P}$ of solution $y$, we obtain an integral equation

$$
(b-a) \int_{a}^{x_{T P}} f\left(s, y(s), y^{\prime}(s)\right) \mathrm{d} s=(B-A)+\int_{a}^{b}\left(\int_{a}^{z} f\left(s, y(s), y^{\prime}(s)\right) \mathrm{d} s\right) \mathrm{d} z .
$$

Changing the order of integration in the integral on the right side (Fubini's theorem), we obtain (2.1).

Corollary 1. Let $y$ be the solution of the problem (1.1), (1.2) with $A=B$. Then the solution $y$ has an unique turning point $x_{T P}$.

Proof. The mean value theorem for integrals implies the existence of $\lambda \in(a, b]$ such that

$$
(b-a) \int_{a}^{\lambda} f\left(s, y(s), y^{\prime}(s)\right) \mathrm{d} s=\int_{a}^{b} f\left(s, y(s), y^{\prime}(s)\right)(b-s) \mathrm{d} s
$$

holds. Moreover, from the assumption $f<0$ it follows that the $\lambda$ is unique and $\lambda \in(a, b)$. Thus, $\lambda=x_{T P}$.

Remark 1. If $A \neq B$, then the existence of a turning point is not guaranteed. Indeed, consider the trivial problem $y^{\prime \prime}-1=0$ subject to the boundary conditions $(1.2)$ on the interval $[0,1]$. The solution of this boundary value problem is

$$
y(x)=\frac{x^{2}}{2}+\left(B-A-\frac{1}{2}\right) x+A
$$

with a turning point $x_{T P}=\frac{1}{2}-(B-A)$. Hence, for $|B-A| \geq \frac{1}{2}$ there is no turning point of solution in the interval $(0,1)$.

\subsection{The existence of solutions}

In this subsection, we prove the existence of solutions for problem (1.1), (1.2). To do so we make the following assumption about the growth rate of $f\left(x, y, y^{\prime}\right)$ with respect to the variable $y^{\prime}$.

(H) Let $f$ satisfy the Nagumo condition, that is, for each $K>0$ there exists a continuous function $h_{K}:[0, \infty) \rightarrow\left[a_{K}, \infty\right)$ with $a_{K}>0$ and

$$
\int_{0}^{\infty} \frac{s \mathrm{~d} s}{h_{K}(s)}=\infty
$$

such that for all $y,|y| \leq K$ all $x \in[a, b]$ and all $y^{\prime} \in \mathbb{R}$ is

$$
\left|f\left(x, y, y^{\prime}\right)\right| \leq h_{K}\left(\left|y^{\prime}\right|\right) \text {. }
$$


The growth condition $(H)$ on $f\left(x, y, y^{\prime}\right)$ along with the assumed existence of solutions $\alpha(x), \beta(x)(\alpha \leq \beta$ on $[a, b])$ of certain types of differential inequalities plays an important role in demonstrating the existence of solutions of boundary value problems not only for second-order differential equations, see e.g. [3, 4, 5]. The most general type of the functions $f$, satisfying the Nagumo condition, are the following:

$$
\left|f\left(x, y, y^{\prime}\right)\right|=\mathcal{O}\left(\left|y^{\prime}\right|^{2}\right) \quad \text { as }\left|y^{\prime}\right| \rightarrow \infty
$$

for all $(x, y) \in[a, b] \times[\alpha(x), \beta(x)]$. The importance of Nagumo condition is that it provides a priori bounds on the first derivative of the possible solutions of the problem under consideration lying between $\alpha$ and $\beta$ on $[a, b]$. More concretely, $\left|y^{\prime}\right| \leq L$ where

$$
\int_{\mu}^{L} \frac{s \mathrm{~d} s}{h_{K}(s)}>\max _{x \in[a, b]} \beta(x)-\min _{x \in[a, b]} \alpha(x)
$$

(see, e.g. [2]) with

$$
K=\max _{x \in[a, b]}\{|\beta(x)|,|\alpha(x)|\} \text { and } \mu=\frac{1}{b-a} \max \{|\beta(b)-\alpha(a)|,|\beta(a)-\alpha(b)|\} .
$$

A comprehensive overview and applications of the lower and upper solutions method can be found in [2] and [3].

Let us denote

$$
\Omega=[a, b] \times[\alpha(x), \beta(x)] \times\left[\min \left\{-L, \alpha^{\prime}(x), \beta^{\prime}(x)\right\}, \max \left\{L, \alpha^{\prime}(x), \beta^{\prime}(x)\right\}\right] .
$$

Theorem 2. Let $f$ be a continuous function such that

$$
m \leq f\left(x, y, y^{\prime}\right) \leq M<0
$$

for every $\left(x, y, y^{\prime}\right) \in \Omega$, where $m \neq M$ are the negative real constants, $\alpha(x)=$ $y_{m}(x)$ and $\beta(x)=y_{M}(x)$ are the solutions of the problem $y^{\prime \prime}+m=0$ and $y^{\prime \prime}+M=0$, respectively, satisfying the boundary conditions $y_{i}(a)=A, y_{i}(b)=$ $B, i=m, M$.

Let $f$ satisfy the Nagumo condition $(\mathrm{H})$. Then the problem (1.1), (1.2) has at least one solution $y$ lying between $y_{m}$ and $y_{M}$ on $[a, b]$.

Proof. We use the method of lower and upper solutions. As usual (see e.g. [3]), a function $\alpha \in C^{2}([a, b])$ is a lower solution for (1.1), (1.2) if $\alpha^{\prime \prime}+f\left(x, \alpha, \alpha^{\prime}\right) \geq$ 0 and $\alpha(a) \leq A, \alpha(b) \leq B$. An upper solution $\beta \in C^{2}([a, b])$ is defined analogously by reversing the inequalities. If $\alpha \leq \beta$ on $[a, b]$ then, under the assumption $(H)$, there exists a solution $y$ of problem (1.1), (1.2) satisfying $\alpha \leq y \leq \beta$ on $[a, b]$.

For $\alpha(x)=y_{m}(x)$ and $\beta(x)=y_{M}(x)$ we have

$$
\begin{gathered}
\alpha^{\prime \prime}(x)+f\left(x, \alpha(x), \alpha^{\prime}(x)\right)=-m+f\left(x, \alpha(x), \alpha^{\prime}(x)\right) \geq 0, \\
\beta^{\prime \prime}(x)+f\left(x, \beta(x), \beta^{\prime}(x)\right)=-M+f\left(x, \beta(x), \beta^{\prime}(x)\right) \leq 0
\end{gathered}
$$

and $\alpha, \beta$ satisfy the boundary conditions required for the lower and upper solutions for (1.1), (1.2). It remains to prove that $\alpha \leq \beta$ on $[a, b]$. 
Let $\gamma(x)=\beta(x)-\alpha(x)$. Then $\gamma^{\prime \prime}(x)=(m-M)<0$ and $\gamma(a)=\gamma(b)=0$. Thus, the function $\gamma$ is positive on $(a, b)$ and on the basis of the method of lower and upper solutions there exists a solution $y$ of the problem (1.1), (1.2) such that

$$
y_{m}(x) \leq y(x) \leq y_{M}(x)
$$

for every $x \in[a, b]$ where

$$
\begin{aligned}
& y_{i}(x)=-\frac{x^{2} i}{2}+c_{1} x+c_{2}, \quad i=m, M, \\
& c_{1}=\frac{k_{2}-k_{1}}{b-a}, \quad c_{2}=\frac{k_{1} b-k_{2} a}{b-a}, \quad k_{1}=A+\frac{a^{2} i}{2}, \quad k_{2}=B+\frac{b^{2} i}{2} .
\end{aligned}
$$

\section{An Estimate of a Turning Point Location}

In this section we state and prove a theorem which is the main result of article concerning the estimate of location of a turning point $x_{T P}$ of solution $y$ of the problem (1.1), (1.2).

Theorem 3. Let the assumptions of Theorem 2 are fulfilled. Suppose in addition that

$$
m \leq g_{L}(x) \phi_{L}(y) \leq f\left(x, y, y^{\prime}\right) \leq g_{U}(x) \phi_{U}(y) \leq M<0
$$

for every $\left(x, y, y^{\prime}\right) \in \Omega$ with the continuous functions $g_{L}(x), g_{U}(x)$ on the interval $[a, b]$ and with the continuous monotone nondecreasing functions $\phi_{L}(y)>0$, $\phi_{U}(y)>0$ on the interval $\left[\min _{x \in[a, b]} \alpha(x), \max _{x \in[a, b]} \beta(x)\right]$. Let $A=B$.

Then

$$
x_{L} \leq x_{T P} \leq x_{U},
$$

where $x_{L}$ and $x_{U}$ are the solutions of equations

$$
(b-a) \int_{a}^{x_{L}} g_{L}(s) \phi_{L}(\beta(s)) \mathrm{d} s=\int_{a}^{b} g_{U}(s) \phi_{U}(\alpha(s))(b-s) \mathrm{d} s
$$

and

$$
(b-a) \int_{a}^{x_{U}} g_{U}(s) \phi_{U}(\alpha(s)) \mathrm{d} s=\int_{a}^{b} g_{L}(s) \phi_{L}(\beta(s))(b-s) \mathrm{d} s,
$$

respectively, and

$$
x_{U}-x_{L} \leq\left(\frac{m}{M}-\frac{M}{m}\right) \frac{b-a}{2} .
$$

Proof. The relation $x_{L} \leq x_{T P} \leq x_{U}$ can be obtained in the case of nondecreasing $\phi_{L}(y)$ and $\phi_{U}(y)$ as follows:

$$
\begin{aligned}
(b- & -a) \int_{a}^{x_{T P}} f\left(s, y(s), y^{\prime}(s)\right) \mathrm{d} s=\int_{a}^{b} f\left(s, y(s), y^{\prime}(s)\right)(b-s) \mathrm{d} s \\
& \leq \int_{a}^{b} g_{U}(s) \phi_{U}(\alpha(s))(b-s) \mathrm{d} s=(b-a) \int_{a}^{x_{L}} g_{L}(s) \phi_{L}(\beta(s)) \mathrm{d} s .
\end{aligned}
$$


Then

$$
\int_{a}^{x_{T P}} f\left(s, y(s), y^{\prime}(s)\right) \mathrm{d} s \leq \int_{a}^{x_{L}} g_{L}(s) \phi_{L}(\beta(s)) \mathrm{d} s
$$

and in view of

$$
0>f\left(x, y, y^{\prime}\right) \geq g_{L}(x) \phi_{L}(\beta(x))
$$

and

$$
\int_{a}^{x_{T P}}\left[-f\left(s, y(s), y^{\prime}(s)\right)\right] \mathrm{d} s \geq \int_{a}^{x_{L}}\left[-g_{L}(s) \phi_{L}(\beta(s))\right] \mathrm{d} s
$$

it follows that $x_{T P} \geq x_{L}$. We analogously obtain the second part of the relation (3.1) $\left(x_{T P} \leq x_{U}\right)$ by using (2.1) and (3.3).

The estimate (3.4) of the difference between $x_{U}$ and $x_{L}$ results from (3.2) and (3.3) if we put $g_{L}(x) \phi_{L}(y)=m$ and $g_{U}(x) \phi_{U}(y)=M$.

Remark 2. In Theorem 3, we only consider the case of nondecreasing functions $\phi_{L}(y)$ and $\phi_{U}(y)$. The remaining three combinations of monotonicity assumptions on the functions $\phi_{L}(y), \phi_{U}(y)$ can be analyzed analogously to get corresponding to (3.2) and (3.3) equations.

To illustrate how our main result can be used in practice we present an example.

Example 1. Let us consider the nonlinear boundary value problem

$$
y^{\prime \prime}-\left(\frac{\left|y+y^{\prime}\right|}{3+\left(y+y^{\prime}\right)^{2}}\right)^{6}-\cos x=0, \quad y(0)=A=B=y\left(\frac{\pi}{4}\right)
$$

on the interval $\left[0, \frac{\pi}{4}\right]$. The functions $g_{L}, g_{U}, \phi_{L}$ and $\phi_{U}$ may be chosen as

$$
\begin{gathered}
g_{L}(x)=-\frac{27}{46656}-\cos x, \quad \phi_{L}(y) \equiv 1 \quad\left(m=-\frac{46683}{46656}=-1.0005787\right), \\
g_{U}(x)=-\cos x, \quad \phi_{U}(y) \equiv 1 \quad\left(M=-\frac{\sqrt{2}}{2}=-0.707106781 \ldots\right)
\end{gathered}
$$

for $\left(x, y, y^{\prime}\right) \in\left[0, \frac{\pi}{4}\right] \times \mathbb{R}^{2} \supset \Omega$. The existence of solution (see Fig. 1) follows from Theorem 2 .

Further, for a lower bound $x_{L}$ of the unique turning point $x_{T P}$ of solution for the considered boundary value problem we obtain from (3.2) the equation

$$
\frac{\pi}{4} \int_{0}^{x_{L}}\left(\frac{27}{46656}+\cos s\right) \mathrm{d} s=\int_{0}^{\frac{\pi}{4}} \cos s\left(\frac{\pi}{4}-s\right) \mathrm{d} s
$$

and analogously from (3.3) for an upper bound $x_{U}$ we have the equation

$$
\frac{\pi}{4} \int_{0}^{x_{U}} \cos s \mathrm{~d} s=\int_{0}^{\frac{\pi}{4}}\left(\frac{27}{46656}+\cos s\right)\left(\frac{\pi}{4}-s\right) \mathrm{d} s .
$$

Hence,

$$
\frac{27}{46656} x_{L}+\sin x_{L}=0.372923228 \ldots \quad \text { and } \quad \sin x_{U}=0.373150485 \ldots
$$




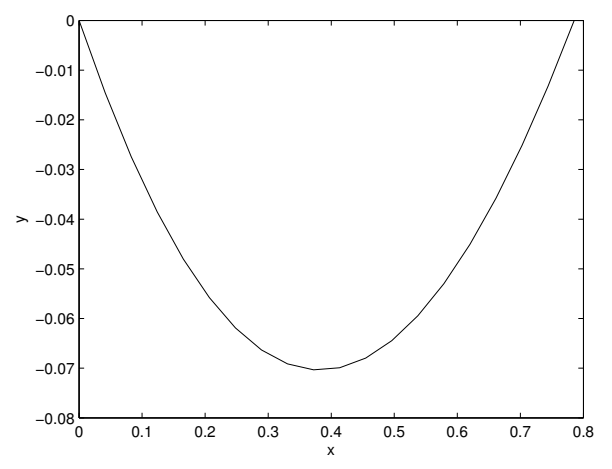

Figure 1. Solution of (3.5) for $A=B=0$.

The values of $x_{L}$ and $x_{U}$ are found by solving these equations separately; the root $x_{L}$ is computed by using numerical method. Thus we obtain the estimate for $x_{T P}$ :

$$
0.38191934169462 \ldots \leq x_{T P} \leq 0.38240246919875 \ldots
$$

The following is MATLAB code that graphically solves Example 1 (Fig. 1):

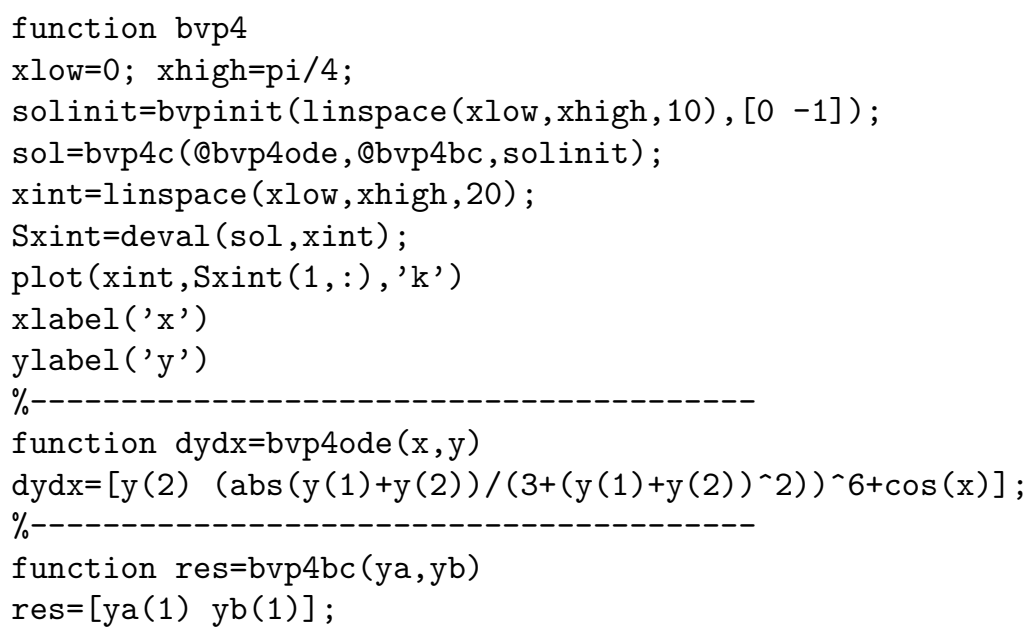

\section{Conclusions}

The techniques for finding the points at which the solution of differential equation attains its local extrema (turning points) provide an additional useful tool to test the validity of the observation-based models.

In this short paper, we focused on the calculation of turning point location for the convex solutions of second-order ordinary differential equation subject to the Dirichlet boundary conditions. 
We have first proved the existence of solutions of a parabola shape (a $U$ shape) by using the method of lower and upper solutions. Then we have derived the integral formulas for calculating the lower and upper bounds of the turning point location without needing to know the exact solution of the boundary value problem under consideration. The efficiency of the proposed approach was demonstrated by the example.

In future research it will be interesting to examine the solutions with more than one turning point.

\section{Acknowledgments}

The author would like to thank the editor and anonymous reviewers for their helpful comments and suggestions toward improving the quality of this paper.

\section{References}

[1] B. Brighi and J.-D. Hoernel. On the concave and convex solutions of mixed convection boundary layer approximation in a porous medium. Appl. Math. Lett., 19(1):69-74, 2006. http://dx.doi.org/10.1016/j.aml.2005.02.038.

[2] A. Cabada. An overview of the lower and upper solutions method with nonlinear boundary value conditions. Bound. Value Probl., 2011(893753):1-18, 2011. http://dx.doi.org/10.1155/2011/893753.

[3] K.W. Chang and F.A. Howes. Nonlinear Singular Perturbation Phenomena: Theory and Applications. Springer-Verlag, New York, 1984.

[4] P. Hartman. On boundary value problems for systems of ordinary, nonlinear, second order differential equations. Trans. Amer. Math. Soc., 96(3):493-509, 1960. http://dx.doi.org/10.1090/S0002-9947-1960-0124553-5.

[5] C.C. Tisdell and L.H. Tan. On vector boundary value problems without growth restriction. J. Inequal. Pure Appl. Math., 6(137):1-10, 2005. 